\title{
Reply to 'Dissipative feedback does not improve the optimal resolution of incoherent force detection'
}

To the Editor - In our article published in $2012^{1}$, we reported an ultralow-noise hybrid structure that combined a high- $Q$ nanomechanical oscillator made of SiN and a silica microresonator. Using ultrasensitive optical readout of the nanomechanical oscillator's motion by means of the optical microresonator, we performed dissipative feedback to increase the effective bandwidth of the nanomechanical oscillator. We subsequently demonstrated that the application of feedback leads to a substantial decrease of the averaging time required for detecting an incoherent force, whose spectrum largely exceeds the bandwidth over which the high- $Q$ mechanical resonator responds. The required averaging time was experimentally shown to decrease as a function of the feedback gain, and the observations were in agreement with our theoretical model.

Vinante et al. ${ }^{2}$ raised two issues. First, that the signal-to-noise ratio of the forces acting on the mechanical oscillator is not changed by dissipative feedback, a fact that is widely known in the literature. This issue is referenced in our work ${ }^{3}$, and is evidenced by equation (1) and Fig. $4 \mathrm{~g}$ of the paper ${ }^{1}$, where the expected signal was explicitly shown to be independent of the feedback gain.
Second, Vinante et al. suggested that energy is not the only possible force estimator, and that the Wiener theory can provide an optimal estimate by means of appropriate post-processing filtering. In this respect, we observed that in our case feedback can be viewed as an analogous approach to optimize the energy estimator in real time, as also noted by Vinante et al. Consequently, our work does establish feedback as an efficient technique for improving force resolution in ultrasensitive force measurements.

Vinante et al. also suggested that feedback does not affect the optimal force estimate derived from the Wiener filtering theory, which they explicitly assume to be applicable to "most resonator-based systems without the need of additional hardware." It should be noted, however, that it remains unclear whether the implementation of the filter-based technique suggested by Vinante et al. can be straightforwardly applied to ultrasensitive nanomechanical systems, which are subject to increased frequency noise $e^{4}$. As a consequence, the mechanical resonance frequency fluctuates randomly, requiring real-time tracking of the mechanical frequency. We have measured the frequency fluctuations in our hybrid nano-optomechanical system ${ }^{4}$, and have determined that the Wiener filter-based technique would be responsible for a degradation of the force estimate, preventing detection of signals below $10 \%$ of the thermal noise spectral density - a factor of 2.5 above the force signal we measured in our paper ${ }^{1}$.

In conclusion, Vinante et al. are theoretically correct to point out that post-processing filtering techniques are an interesting alternative to feedback for optimizing the force estimate. However, the increased amount of frequency noise widely encountered in nanomechanical resonators may compromise the use of the filter-based technique as a reliable way to optimize force resolution.

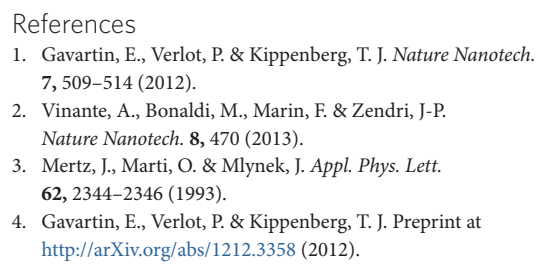

1. Gavartin, E., Verlot, P. \& Kippenberg, T. J. Nature Nanotech. 7, 509-514 (2012).

2. Vinante, A., Bonaldi, M., Marin, F. \& Zendri, J-P. Nature Nanotech. 8, 470 (2013).

3. Mertz, J., Marti, O. \& Mlynek, J. Appl. Phys. Lett. 62, 2344-2346 (1993).

4. Gavartin, E., Verlot, P. \& Kippenberg, T. J. Preprint at http://arXiv.org/abs/1212.3358 (2012).

E. Gavartin, P. Verlot* and T. J. Kippenberg* Ecole Polytechnique Fédérale de Lausanne, EPFL, 1015 Lausanne, Switzerland. *e-mail: pierre.verlot@grenoble.cnrs.fr; tobias.kippenberg@epfl.ch 